\title{
Bell inequalities with no quantum violation and unextendible product bases
}

\author{
R. Augusiak ${ }^{1}$, J. Stasińska ${ }^{2}$, C. Hadley ${ }^{1}$, J. K. Korbicz ${ }^{1}$, M. Lewenstein ${ }^{1,3}$ and A. Acín ${ }^{1,3}$ \\ ${ }^{1}$ ICFO-Institut de Ciències Fotòniques, 08860 Castelldefels (Barcelona), Spain \\ ${ }^{2}$ Grup de Física Teòrica: Informació i Fenòmens Quàntics, \\ Universitat Autònoma de Barcelona, 08193 Bellaterra (Barcelona), Spain and \\ ${ }^{3}$ ICREA-Institució Catalana de Recerca i Estudis Avançats, Lluis Companys 23, 08010 Barcelona, Spain
}

\begin{abstract}
The strength of classical correlations is subject to certain constraints, commonly known as Bell inequalities. Violation of these inequalities is the manifestation of non-locality - displayed, in particular, by quantum mechanics, meaning that quantum mechanics can outperform classical physics at tasks associated with such Bell inequalities. Interestingly, however, there exist situations in which this is not the case. We associate an intriguing class of bound entangled states, constructed from unextendable product bases (UPBs) with a wide family of tasks, for which (i) quantum correlations do not outperform the classical ones but (ii) there exist supraquantum nonsignalling correlations that do provide an advantage.
\end{abstract}

Introduction.-The existence of correlations is an inherent property of composite physical systems and, as such, is fundamental for our understanding of physical phenomena. On the other hand, physical principles impose limits on the correlations between the results of measurements performed on distant systems. If the measurements correspond to spacelike separated events, the observed correlations should obey the principle of no signaling, which prevents any faster-than-light communication among the parties. If the systems are quantum, it should be possible to write the correlations as results of local measurements acting on a global quantum state. Finally, the observed correlations are said to be classical if they are attainable with shared classical randomness. All three kinds of correlations correspond to sets of probabilities of measurement outcomes and, as such, form convex sets, represented schematically in Fig. 1.

Bell was the first to point out that classical correlations (CC) are constrained by certain inequalities (the famous Bell inequalities) [1. Correlations which violate a Bell inequality, and thus do not correspond to any classical model, are known as nonlocal. Bell's theorem guarantees the existence of quantum correlations (QC) that are nonlocal. However, it is known also that there are nonsignaling correlations (NC) which are supraquantum [2], i.e., not attainable by measurements acting on a quantum state, yet violating Bell inequalities.

Apart from its fundamental importance, understanding the relation among the sets of correlations is crucial from a practical point of view, since correlations and application as an information resource. In particular, one of the goals of quantum information theory is to understand when QC give an advantage over CC. For instance, nonlocal QC provide cryptographic security not achievable with classical theory [3, 4]. They can also be used to certify the presence of randomness [5] and outperform CC at communication complexity problems [6].

While QC are in general more powerful than CC, there are some intriguing situations in which $\mathrm{CC}$ and QC perform equally well. This equivalence can be detected by

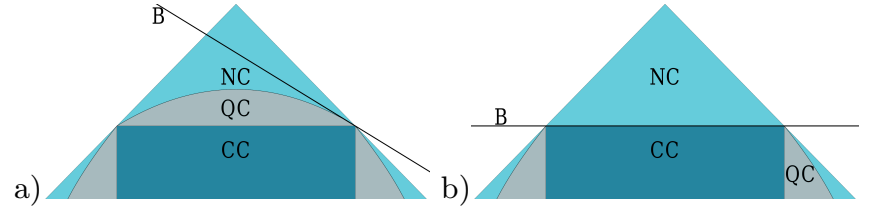

FIG. 1: Schematic depiction of the sets of classical $(C C)$, quantum $(Q C)$ and non-signalling correlations $(N C)$. Tight Bell inequalities correspond to facets of the classical set. $B$ denotes a Bell inequality with no quantum violation which is a) not tight, b) tight. Note that a tight Bell inequality guarantees that a region in which quantum and classical correlations coincide is a facet. A non-tight inequality may define a common region of the classical and quantum sets that does not have maximal dimension.

Bell inequalities which are not violated by QC. The first examples of such inequalities were given in Ref. 7] for two parties. Unfortunately, none of these is tight 8 . The importance of tight Bell inequalities stems from the fact that they correspond to facets of the convex set (polytope) of classical correlations (see Fig. 1) and hence are sufficient to fully characterize it [9]. Multipartite Bell inequalities with no quantum violation were later provided in Ref. [10] and, moreover, shown to be tight for $3 \leq n \leq 7$ parties. Apart from these examples, we know little about information tasks, or equivalently Bell inequalities, where QC do not provide any advantage.

In this Letter, we demonstrate an a priori unexpected relation between such inequalities and unextendable product bases (UPBs) 11. Recall that the latter is a collection of orthogonal product vectors spanning a proper subspace $V$ of some $n$-partite Hilbert space $\mathcal{H}$, such that there does not exist any other product vector in $\mathcal{H}$ orthogonal to $V$. The fundamental physical importance of UPBs stems from the fact that they allow for the construction [11] of one of the first examples of bound (i.e., nondistillable) entangled states-being one of the most intriguing concepts in quantum information theory [12]-and, furthermore, that they give rise to nonlocality without entanglement [13, i.e., the impossibility 
of perfect distinguishability of some orthogonal product states by means of local operations and classical communication.

Below, we prove how UPBs satisfying a given requirement give rise to Bell inequalities without a quantum violation. Our construction covers some of the inequalities previously derived in Ref. [10] and, thus, can lead to tight Bell inequalities. Moreover, the construction can be exploited in the opposite direction: We derive new examples of UPBs from some of the Bell inequalities with no quantum violation from Ref. [10].

The considered scenario consists of $n$ distant observers having access to $n$ systems. Observer $i(i=1, \ldots, n)$ can perform on his system one of $m_{i}$ possible measurements. His choice of measurement is denoted $x_{i}=1, \ldots, m_{i}$, and the obtained result $a_{i}=1, \ldots, r_{i}^{x_{i}}$ with $r_{i}^{x_{i}}$ denoting the number of outcomes the $m_{i}$ th measurement has, while $\mathbf{x}=\left(x_{1}, \ldots, x_{n}\right)$ and $\mathbf{a}=\left(a_{1}, \ldots, a_{n}\right)$ stand for the corresponding vectors. The correlations among the parties are described by the conditional probability $p(\mathbf{a} \mid \mathbf{x})$.

Consider a linear combination of these probabilities defined by a $2 n$-index tensor $T_{\mathbf{x}, \mathbf{a}}, \sum T_{\mathbf{x}, \mathbf{a}} p(\mathbf{a} \mid \mathbf{x})$. It leads to the Bell inequality $\sum_{\mathbf{x}, \mathbf{a}} T_{\mathbf{x}, \mathbf{a}} p(\mathbf{a} \mid \mathbf{x}) \leq \beta_{\mathrm{C}}$, where $\beta_{\mathrm{C}}\left(\beta_{\mathrm{Q}}\right.$, $\left.\beta_{\mathrm{N}}\right)$ is its maximal value for $\mathrm{CC}(\mathrm{QC}, \mathrm{NC})$. It is nontrivial if it is not an inequality for general non-signalling correlations, i.e., $\beta_{\mathrm{N}}>\beta_{\mathrm{C}}$, while it is violated by $\mathrm{QC}$ if $\beta_{\mathrm{Q}}>\beta_{\mathrm{C}}$. Any Bell inequality can also be seen as a nonlocal game, in which the parties are given the input $\mathbf{x}$ and have to produce the output $\mathbf{a}$, in a distributed manner, all the possibilities being weighted by the tensor $T_{\mathbf{x}, \mathbf{a}}$. The maximum values of the inequalities give the optimal winning probability for the different sets of correlations.

From a UPB to Bell inequalities. - Consider an $n$ partite product Hilbert space $\mathcal{H}=\otimes_{i=1}^{n} \mathbb{C}^{d_{i}}$ and a set $\mathcal{S}$ of orthogonal product vectors from $\mathcal{H}: \mathcal{S}=\left\{\left|\psi_{j}^{(1)}\right\rangle \otimes\right.$ $\left.\ldots \otimes\left|\psi_{j}^{(n)}\right\rangle\right\}_{j=1}^{|\mathcal{S}|}$, with $\left|\psi_{j}^{(i)}\right\rangle \in \mathbb{C}^{d_{i}}$. The $|\mathcal{S}|$ local states for each party constitute $n$ sets, denoted by $\mathcal{S}^{(i)}$.

We partition each $\mathcal{S}^{(i)}$ into disjoint subsets $\mathcal{S}_{k}^{(i)}$ such that all vectors forming a particular subset are mutually orthogonal. Each $\mathcal{S}_{k}^{(i)}$ defines a measurement, while the different vectors within every such subset are associated to the measurement outcomes. In order to remove the ambiguity in splitting the local sets $\mathcal{S}^{(i)}$ into subsets, we restrict ourselves to sets $\mathcal{S}$ having the property that no two vectors belonging to different subsets $\mathcal{S}_{k}^{(i)}$ are orthogonal. Below we refer to this property as $(\mathrm{P})$. This constraint is automatically satisfied in the case of qubits.

It is straightforward to assign to each vector from $\mathcal{S}$ a conditional probability $p\left(\boldsymbol{a}_{j} \mid \boldsymbol{x}_{j}\right)$ : the measurement by the observer $i$ is given by the index $k$, enumerating the subset $\mathcal{S}_{k}^{(i)}$ to which $\left|\psi_{j}^{(i)}\right\rangle$ belongs, while the result corresponds to the position of this state within the set. We now consider linear combinations of these conditional probabilities with weights $q_{j}$. The maximum of such a linear combination over all local strategies is
$\beta_{\mathrm{C}}=\max \left\{q_{j}\right\}$. Indeed, due to $(\mathrm{P})$, orthogonality of any two vectors from $\mathcal{S}$ means that at some position they have different vectors from the same local subset. At the level of probabilities, this means that if one of them, say $p\left(\boldsymbol{a}_{j} \mid \boldsymbol{x}_{j}\right)$, equals unity, the rest have to be zero, as they always have at some position the same input but a different output. Then, we get the Bell inequality

$$
\sum_{j} q_{j} p\left(\boldsymbol{a}_{j} \mid \boldsymbol{x}_{j}\right) \leq \max \left\{q_{j}\right\}
$$

from the initial set of orthogonal product vectors $\mathcal{S}$.

It is now easy to prove that all these inequalities are not violated by $\mathrm{QC}$.

Fact 1. Let $\mathcal{S}$ be a set of orthogonal product vectors possessing the property $(P)$. Then for the corresponding Bell inequality (1) it holds that $\beta_{\mathrm{C}}=\beta_{\mathrm{Q}}=\max \left\{q_{i}\right\}$.

Proof. First of all, since the dimension is arbitrary, we can restrict the analysis to projective measurements. Let us assign projectors $P_{j}^{(i)}$ (we enumerate them in the same way as the vectors $\left.\left|\psi_{j}^{(i)}\right\rangle\right)$ to the outcomes of the local observables, and construct the Bell operator

$$
B=\sum_{j=1}^{|\mathcal{S}|} q_{j} \bigotimes_{i=1}^{n} P_{j}^{(i)}
$$

In general, $P_{j}^{(i)}$ may be different from the local vectors of $\mathcal{S}$ and, moreover, they can be degenerate. Nevertheless, they maintain the orthogonality of the local vectors from $\mathcal{S}$. Precisely, any pair of projectors $\otimes_{i=1}^{n} P_{j}^{(i)}$ have at some position local projectors corresponding to the same observable but different outcomes, meaning that all of them are orthogonal. Thus the maximal eigenvalue of $B$ is $\max \left\{q_{i}\right\}$, and hence $\beta_{\mathrm{Q}}=\max \left\{q_{i}\right\}$. I

Our construction offers a systematic and easy way of generating Bell inequalities with no quantum violation from orthogonal product vectors. However, it could be the case that all the derived inequalities are trivial, in the sense of not being violated by any NC. Here is where the concept of a UPB becomes relevant.

Fact 2. If $\mathcal{S}$ is a UPB with the property $(P)$, the resulting Bell inequality (1) with $q_{j}=1$ is violated by $N C$.

Proof. Let $\Pi_{\mathrm{UPB}}$ be a projector onto the subspace of $\mathcal{H}$ spanned by $\mathcal{S}$. Then, the Bell operator $B$ with $q_{j}=1$ and the measurements defined by $\mathcal{S}$ is exactly $\Pi_{\mathrm{UPB}}$.

Consider now the normalized entanglement witness $W=[1 /(|\mathcal{S}|-\epsilon D)]\left(\Pi_{\mathrm{UPB}}-\epsilon \mathbb{1}\right)$ with $D=\operatorname{dim} \mathcal{H}$ and $\epsilon=\min _{\psi_{\text {prod }}}\left\langle\psi_{\text {prod }}\left|\Pi_{\text {UPB }}\right| \psi_{\text {prod }}\right\rangle$. This witness detects the bound entangled state $\varrho=\left(\mathbb{1}-\Pi_{\mathrm{UPB}}\right) /(D-|\mathcal{S}|)$. A direct check shows that $\operatorname{Tr}(B W)=\operatorname{Tr}\left(\Pi_{\mathrm{UPB}} W\right)=$ $|\mathcal{S}|(1-\epsilon) /(|\mathcal{S}|-\epsilon D)$, which is greater than one (in this case $\beta_{C}=\beta_{Q}=1$ ) whenever $|\mathcal{S}|>\epsilon D$. The latter, however, follows from the very definition of $W$. Consequently, $W$ violates the Bell inequality resulting from $\mathcal{S}$. This completes the proof, as local measurements acting on a witness give raise to NC (see, e.g., Refs. [14, 15]). 
Our construction, then, shows how to derive nontrivial Bell inequalities with no quantum violation from any UPB with property $(\mathrm{P})$. As mentioned, this property is always satisfied in the case of qubits. For two parties there exists no qubit UPB. Moving to three parties, it was shown in Ref. [16], that by local unitaries and permutations of particles, all UPBs can be brought to: $\mathcal{S}=\left\{|000\rangle,\left|1 e_{2} e_{3}\right\rangle,\left|e_{1} 1 e_{3}^{\perp}\right\rangle,\left|e_{1}^{\perp} e_{2}^{\perp} 1\right\rangle\right\}$ with $\left|e_{i}\right\rangle \neq|0\rangle,|1\rangle$ and $\left\langle e_{i}^{\perp} \mid e_{i}\right\rangle=0(i=1,2,3)$. Following the above rules, at each site we can define two subsets of mutually orthogonal vectors, namely, $\mathcal{S}_{0}=\{|0\rangle,|1\rangle\}$ and $\mathcal{S}_{1}^{(i)}=\left\{\left|e_{i}\right\rangle,\left|e_{i}^{\perp}\right\rangle\right\}$. Then, we assign to each element in the UPB the following probabilities: $|000\rangle \rightarrow$ $p(000 \mid 000),\left|1 e_{2} e_{3}\right\rangle \rightarrow p(100 \mid 011),\left|e_{1} 1 e_{3}^{\perp}\right\rangle \rightarrow p(011 \mid 101)$, and $\left|e_{1}^{\perp} e_{2}^{\perp} 1\right\rangle \rightarrow p(111 \mid 110)$. Adding them, we obtain the inequality $p(000 \mid 000)+p(100 \mid 011)+p(011 \mid 101)+$ $p(111 \mid 110) \leq 1$ with $\beta_{\mathrm{Q}}=\beta_{\mathrm{C}}=1$. This inequality, previously derived in Ref. [17], is one of the tight inequalities studied in Ref. [10]. This shows that our construction can lead to tight Bell inequalities with no quantum violation. In Ref. [18, the above UPB was generalized to an arbitrary odd number of qubits. We have checked that the corresponding inequality for $n=5$, which is not the same as the five-party Bell inequality of [10, is not tight.

Moving to dimensions larger than two, there already exist UPBs for two parties. Although, as explained later, none of them has property $(\mathrm{P})$, there do exist examples for more than two parties with this property, such as the UPB of Ref. 19. We applied our construction to these states in the case of four three-dimensional system. Unfortunately, the resulting inequality is not tight.

From a Bell inequality to UPB. - Clearly, the above procedure can be applied in reverse: given a Bell inequality (1), one can derive, following analogous rules, a set of product vectors. The number of different inputs at each position gives the number of different local subsets, while the number of different outputs corresponding to a particular input gives the number of elements of the corresponding subset. The maximal number of different outputs at the $i$ th position gives the dimension of the local Hilbert space $d_{i}$. Note that in the general case, the derived vectors are not necessarily orthogonal. In what follows, we consider the set of Bell inequalities with no quantum violation given in [10. These inequalities are such that the derived product vectors are orthogonal and naturally possesses the property $(\mathrm{P})$. Remarkably, as we will see shortly, they define a new class of UPBs.

The explicit form of these inequalities for odd $n$ reads

$$
\sum_{k=0}^{(n-1) / 2} \sum_{i_{1}<\ldots<i_{2 k}=1}^{n} D_{i_{1} \ldots i_{2 k}} p(\mathbf{0} \mid \mathbf{0}) \leq 1,
$$

while for even $n$,

$$
\sum_{k=0}^{(n-2) / 2} \sum_{i_{1}<\ldots<i_{2 k}=2}^{n} D_{i_{1} \ldots i_{2 k}}[p(\mathbf{0} \mid \mathbf{0})+p(0 \ldots 01 \mid 10 \ldots 0)] \leq 1 .
$$

Here $\mathbf{0}=(0, \ldots, 0)$ and $D_{i_{1}, \ldots, i_{k}}$ flips $(0 \leftrightarrow 1)$ inputs and outputs at positions $i_{1}, \ldots, i_{k}$ and $i_{1}-1, \ldots, i_{k}-1$ (if $i_{j}=1$ then $i_{j}-1=n$ ), respectively.

We now derive the product vectors corresponding to these inequalities for arbitrary $n$. Note that all terms in Eqs. (3) and (4) have at each position two possible incomes and outcomes. Thus, at each site we can define a pair of two-element sets and, without any loss of generality, we can take them to be equal for all sites; say $\mathcal{S}_{0}=\{|0\rangle,|1\rangle\}$ and $\mathcal{S}_{1}=\left\{|e\rangle,\left|e^{\perp}\right\rangle\right\}$ with $|e\rangle \neq|0\rangle,|1\rangle$.

Let $V$ denote a unitary operation such that $V|0\rangle=|e\rangle$ and $V|1\rangle=\left|e^{\perp}\right\rangle$, while $\sigma_{x}$, the standard Pauli matrix flipping $|0\rangle \leftrightarrow|1\rangle$. Then, the $2^{n-1}$ product vectors derived from (3) and (4) can be written as

$$
\begin{array}{r}
V_{i_{1}} \ldots V_{i_{k}} \sigma_{i_{1}-1} \ldots \sigma_{i_{k}-1}|0\rangle^{\otimes n} \\
i_{1}<\ldots<i_{k}=1, \ldots, n, \quad k=0,2,4, \ldots, n-1
\end{array}
$$

and

$$
\begin{array}{r}
V_{i_{1}} \ldots V_{i_{k}} \sigma_{i_{1}-1} \ldots \sigma_{i_{k}-1}|0\rangle^{\otimes n} \\
V_{1} V_{i_{1}} \ldots V_{i_{k}} \sigma_{i_{1}-1} \ldots \sigma_{i_{k}-1} \sigma_{n}|0\rangle^{\otimes n} \\
i_{1}<\ldots<i_{k}=2, \ldots, n, \quad k=0,2,4, \ldots, n-2,
\end{array}
$$

respectively. For $n=3$ we recover the four-element threequbit Shifts UPB [11. Notice that the freedom in choosing the local sets allows one to obtain more general UPBs.

We are now ready to prove the following statement.

Fact 3. The vectors (5) and (6), form an n-qubit UPB. Proof. Our proof consists of two steps. First we show that for any $n$, the above vectors can be generated from the Shifts UPB by a recursive protocol. Then we prove that this protocol preserves the property of being UPB.

Let us start with the case of odd $n$. We denote the set of vectors in Eq. (5) by $U_{1}$ and divide it into two subsets $U_{1}^{(i)}(i=1,2)$ consisting of vectors with the first qubit from $\mathcal{S}_{i-1}$. Then, we create another group of vectors $U_{2}$ by switching the last qubit of $U_{1}$ to the orthogonal one (henceforth called orthogonalization), and divide $U_{2}$ into two subsets $U_{2}^{(i)}(i=1,2)$ in the same way as $U_{1}$. Finally, direct algebra shows that the following set of vectors

$$
|0\rangle \otimes U_{1}^{(1)}, \quad|1\rangle \otimes U_{2}^{(2)}, \quad|e\rangle \otimes U_{2}^{(1)}, \quad\left|e^{\perp}\right\rangle \otimes U_{1}^{(2)},
$$

is exactly the same as the vectors in 6 with $n+1$ parties.

Almost exactly the same procedure produces $(n+1)$ partite vectors (5) from $n$-partite set with even $n$. The only difference is that to obtain $U_{2}$ from $U_{1}$ one has to orthogonalize the penultimate qubit and apply the transformation $|0\rangle \leftrightarrow\left|e^{\perp}\right\rangle$ and $|1\rangle \leftrightarrow|e\rangle$ to the last one.

Having established the recursive procedure generating vectors (5) and (6) from the Shifts UPB, we now show that it preserves the UPB property. First, let us prove that all the vectors (7) are orthogonal. It suffices to prove that vectors from $U_{2}$ are orthogonal, $U_{1}^{(1)} \perp U_{2}^{(1)}$, and $U_{1}^{(2)} \perp U_{2}^{(2)}$ (notice that already $U_{1}^{(1)} \perp U_{1}^{(2)}$ ). The first 
condition is satisfied due to the fact that $U_{2}$ is obtained from $U_{1}$ by application of the above local transformations. A direct check shows that they map a set of orthogonal vectors onto another set of orthogonal vectors.

The proof of the remaining two conditions is more involved. Nevertheless, it suffices to consider the odd- $n$ case, since the proof for the even $n$ goes along almost the same lines. To this end, notice that the last qubit of the vectors in $U_{1}^{(1)}$ is either $|0\rangle$ or $|e\rangle$ (cf. 77)). Thus, their orthogonality comes from the first $n-1$ qubits. This, together with the fact that $U_{2}^{(1)}$ is obtained from $U_{1}^{(1)}$ by orthogonalizing the last qubit, imply that any vector from $U_{1}^{(1)}$ is orthogonal to $U_{2}^{(1)}$ and hence $U_{1}^{(1)} \perp U_{2}^{(1)}$. Exactly the same reasoning allows one to conclude that $U_{1}^{(2)} \perp U_{2}^{(2)}$. The only difference is that the last qubit of $U_{1}^{(2)}$ is either $|1\rangle$ or $\left|e^{\perp}\right\rangle$ (also not orthogonal).

Finally, we show that there does not exist any product vector orthogonal to the set (7). For this purpose, assume the contrary and write the vector orthogonal to (7) as $|\psi\rangle=|x\rangle|\widetilde{\psi}\rangle$ with $|x\rangle$ and $|\widetilde{\psi}\rangle$ denoting the first qubit and the product state of the remaining $n-1$ qubits, respectively. If $|x\rangle$ belongs to one of the sets $\mathcal{S}_{i}(i=0,1)$, say $\mathcal{S}_{0}$, then $|\widetilde{\psi}\rangle$ has to be orthogonal to either $U_{1}$ or $U_{2}$, depending on whether $|x\rangle=|0\rangle$ or $|x\rangle=|1\rangle$. If $|x\rangle \notin \mathcal{S}_{i}$ $(i=0,1)$, then $|\widetilde{\psi}\rangle$ must be orthogonal to both UPBs $U_{i}$. Both situations lead to a contradiction meaning that the above construction preserves the UPB property.

Conclusions. - Nontrivial Bell inequalities lacking a quantum violation are rare and intriguing objects meriting further investigation. We have demonstrated here a systematic way to derive inequalities of this type with the property $\beta_{\mathrm{C}}=\beta_{\mathrm{Q}}<\beta_{\mathrm{N}}$ from UPBs, themselves an important concept in the theory of entanglement. We have furthermore shown that the construction may be applied in the reverse direction and have provided new examples of UPBs from existing Bell inequalities.

These findings are strongly related to recent work on the relationship between QC and multipartite versions of Gleason's theorem [14, 15. Indeed, the generalisation of this theorem to the case of distant observers leads to correlations that can be written as local measurements acting on entanglement witnesses. This set is equivalent to the set of QC in the bipartite case [14, 15. However, this equivalence does not hold for three parties 15. Here, we generalize this result to any Bell scenario in which one is able to build, by using our procedure, a non-trivial inequality from some UPB satisfying $(\mathrm{P})$. On the other hand, our results imply that there are no bipartite UPBs with the property $(\mathrm{P})$, as otherwise there would exist a bipartite witness violating a Bell inequality beyond the quantum bound, contradicting the results of [14, 15].

The connection between product vectors and Bell inequalities introduced here opens new perspectives. For instance, it is worth investigating whether sets of orthogonal product vectors with $(\mathrm{P})$, which are not UPBs can lead to novel Bell inequalities. Although all of them lack a quantum violation, it is unclear whether they are nontrivial, i.e., violated by some nonsignalling correlations. In this direction, we prove the following:

Fact 4. Let $\mathcal{S}$ be a completable set of orthogonal product vectors with the property $(P)$. Then the corresponding Bell inequality (1) is not violated by any $N C$ represented by entanglement witnesses.

Proof. Let $\mathcal{S}^{\perp}$ denote the set of product vectors completing $\mathcal{S}$ to the full basis in $\mathcal{H}$. Then consider the Bell inequality derived from $\mathcal{S}$ and the Bell operator $B$ representing it (cf. 2)]. By $\Pi$ let us now denote the separable projector onto the support of $B$. The latter can act on a Hilbert space of dimension larger than $\operatorname{dim} \mathcal{H}$, but, since $\mathcal{S}$ is completable, there exists a separable projector $\Pi^{\perp}$ such that $\Pi+\Pi^{\perp}=\mathbb{1}$. Then, for any normalized witness $\operatorname{Tr}(B W) \leq \max \left\{q_{i}\right\} \operatorname{Tr}(\Pi W)=\max \left\{q_{i}\right\} \operatorname{Tr}\left[\left(\mathbb{1}-\Pi^{\perp}\right) W\right]$. As $\Pi^{\perp}$ is separable and $W$ is normalized, $0 \leq \operatorname{Tr}\left(W \Pi^{\perp}\right)$ and hence $\operatorname{Tr}(B W) \leq \max \left\{q_{i}\right\}$.

Finally it would be of interest to understand when this construction leads to tight Bell inequalities, and if the ability to do so may be inferred from some properties of the set of product states. From a more general perspective, it remains an open question as to whether there exist bipartite Bell inequalities without a quantum violation.

Acknowledgements. We thank M. Piani, G. Prettico, and A. Winter for discussions. This work was supported by EU projects AQUTE, NAMEQUAM, Q-Essence, and QCS, ERC Grants QUAGATUA and PERCENT, Spanish MINCIN projects FIS2010-14830, FIS2008-00784, FIS2008-01236, FPU AP2008-03043, and QOIT, Generalitat de Catalunya, and Caixa Manresa.

[1] J. S. Bell, Physics (Long Island City, N.Y.) 1, 195 (1964).

[2] S. Popescu and D. Rohrlich, Found. Phys. 24, 379 (1994).

[3] A. K. Ekert, Phys. Rev. Lett. 67, 661 (1991).

[4] A. Acín et al., Phys. Rev. Lett. 98, 230501 (2007).

[5] S. Pironio et al., Nature 464, 1021 (2010); R. Colbeck, PhD Thesis, University of Cambridge, 2007; R. Colbeck and A. Kent, J. Phys. A 44 (9), 095305 (2011).

[6] H. Buhrman et al., Rev. Mod. Phys. 82, 665 (2010).

[7] N. Linden et al., Phys. Rev. Lett. 99, 180502 (2007).

[8] M. L. Almeida, private communication.

[9] B. Grünbaum, Convex polytopes (Springer, New York, 2003).

[10] M. L. Almeida et al., Phys. Rev. Lett. 104, 230404 (2010).

[11] C. H. Bennett et al., Phys. Rev. Lett. 82, 5385 (1999).

[12] M. Horodecki, P. Horodecki, and R. Horodecki, Phys. Rev. Lett. 80, 5239 (1998).

[13] C. H. Bennett et al., Phys. Rev. A 59, 1070 (1999).

[14] H. Barnum et al., Phys. Rev. Lett. 104, 140401 (2010).

[15] A. Acín et al., Phys. Rev. Lett. 104, 140404 (2010).

[16] S. B. Bravyi, Quant. Inf. Proc. 3, 309 (2004).

[17] C. Śliwa, Phys. Lett. A 317, 165 (2003). 
[18] D. P. DiVincenzo et al., Comm. Math. Phys. 238, 379 (2003).

[19] J. Niset and N. J. Cerf, Phys. Rev. A 74, 052103 (2006). 